\title{
Implementation of a cardiac PET stress program: comparison of outcomes to the preceding SPECT era
}

\author{
Stacey Knight,, ${ }^{1,2}$ David B. Min, ${ }^{1}$ Viet T. Le, ${ }^{1,3}$ Kent G. Meredith, ${ }^{1}$ Ritesh Dhar, ${ }^{1}$ Santanu Biswas, ${ }^{1}$ \\ Kurt R. Jensen, ${ }^{1}$ Steven M. Mason, ${ }^{1}$ Jon-David Ethington, ${ }^{1}$ Donald L. Lappe, ${ }^{1,2}$ \\ Joseph B. Muhlestein, ${ }^{1,2}$ Jeffrey L. Anderson, ${ }^{1,2}$ Kirk U. Knowlton, ${ }^{1}$ \\ IIntermountain Heart Institute, Intermountain Medical Center, Murray, Utah, USA. ²Department of Internal Medicine, \\ University of Utah, Salt Lake City, Utah, USA. ${ }^{3}$ Rocky Mountain University of Health Professions Physician Assistant \\ Program, Provo, Utah, USA.
}

BACKGROUND. Cardiac positron emission testing (PET) is more accurate than single photon emission computed tomography (SPECT) at identifying coronary artery disease (CAD); however, the $\mathbf{2}$ modalities have not been thoroughly compared in a real-world setting. We conducted a retrospective analysis of 60-day catheterization outcomes and 1-year major adverse cardiovascular events (MACE) after the transition from a SPECT- to a PET-based myocardial perfusion imaging (MPI) program.

METHODS. MPI patients at Intermountain Medical Center from January 2011-December 2012 (the SPECT era, $n=6,777$ ) and January 2014-December 2015 (the PET era, $n=7,817$ ) were studied. Outcomes studied were 60-day coronary angiography, high-grade obstructive CAD, left main/severe 3-vessel disease, revascularization, and 1-year MACE-revascularization (MACE-revasc; death, myocardial infarction [MI], or revascularization $>60$ days).

RESULTS. Patients were $\mathbf{6 4} \pm \mathbf{1 3}$ years old; $\mathbf{5 4 \%}$ were male and $\mathbf{9 0 \%}$ were of European descent; and $\mathbf{5 7 \%}$ represented a screening population (no prior MI, revascularization, or CAD). During the PET era, compared with the SPECT era, a higher percentage of patients underwent coronary angiography (13.2\% vs. 9.7\%, $P<0.0001$ ), had high-grade obstructive CAD (10.5\% vs. $6.9 \%, P<0.0001)$, had left main or severe 3 -vessel disease (3.0\% vs. $2.3 \%, P=0.012$ ), and had coronary revascularization ( $56.7 \%$ vs. $47.1 \%, P=0.0001$ ). Similar catheterization outcomes were seen when restricted to the screening population. There was no difference in 1-year MACE-revasc (PET [5.8\%] vs. SPECT [5.3\%], $P=0.31)$.

CONCLUSIONS. The PET-based MPI program resulted in improved identification of patients with high-grade obstructive $C A D$, as well as a larger percentage of revascularization, thus resulting in fewer patients undergoing coronary angiography without revascularization.

FUNDING. This observational study was funded using internal departmental funds.

Conflict of interest: The authors have declared that no conflict of interest exists.

Submitted: March 7, 2018

Accepted: April 4, 2018

Published: May 3, 2018

Reference information: JCI Insight. 2018;3(9):e120949. https://doi.org/10.1172/jici. insight.120949.

\section{Introduction}

Myocardial perfusion imaging (MPI) is utilized to determine the presence and extent of ischemic coronary artery disease (CAD). For over 30 years, single photon emission computed tomography (SPECT) has been the primary modality for MPI. Positron emission tomography (PET) scanning has replaced or supplemented SPECT imaging in some centers, but it has not been utilized as broadly as SPECT. However, more centers are considering a switch to PET MPI, given the greater availability of ${ }^{82} \mathrm{Sr} /{ }^{82} \mathrm{Rb}$ generators negating the requirement of an onsite cyclotron. Moreover, PET MPI requires a lower dose of radiation exposure to the patient (1), PET requires shorter time to obtain the images (2), PET has the ability to quantify myocardial blood flow (3), and PET has improved resolution, which has been shown to increase accuracy over SPECT imaging (4-10). These advantages have prompted the American Society of Nuclear Cardiology and Society of Nuclear Medicine and Molecular Imaging to release a joint position paper highlighting the properties of 
Table 1. Demographic and clinical characteristics of the patients for the SPECT and PET eras

\begin{tabular}{|c|c|c|c|}
\hline & SPECT era & PET era & \\
\hline Patient characteristic & $n=6777$ & $n=7817$ & $P$ value \\
\hline Age, mean (SD) years & $63.8(12.8)$ & $64.1(12.7)$ & 0.12 \\
\hline European descent, no. (\%) & 5965 (88.0\%) & 7180 (91.9\%) & $<0.0001$ \\
\hline BMI, mean (SD) & $31.2(7.2)$ & $31.7(7.4)$ & 0.0001 \\
\hline Hypertension, no. (\%) & $4,208(62.1 \%)$ & 4,932 (63.1\%) & 0.21 \\
\hline Family history of CVD, no. (\%) & $361(55.5 \%)$ & $4,540(58.1 \%)$ & 0.002 \\
\hline Smoking history, no. (\%) & $2,502(36.9 \%)$ & 2,916 (37.3\%) & 0.06 \\
\hline Prior MI, no. (\%) & $1,592(23.5 \%)$ & $1,662(21.3 \%)$ & 0.001 \\
\hline Heart failure, no. (\%) & $1,380(20.4 \%)$ & $1,736(22.2 \%)$ & 0.01 \\
\hline Prior CAD, no. (\%) & $2,504(36.9 \%)$ & $3,046(39.0 \%)$ & 0.01 \\
\hline Prior stress test, no. (\%) & $1,227(18.1 \%)$ & 1,339 (17.1\%) & 0.12 \\
\hline \multicolumn{4}{|l|}{ Prior medications, no. (\%) } \\
\hline ACE-I or ARB & $1,342(19.8 \%)$ & 2,467 (31.6\%) & $<0.0001$ \\
\hline Aspirin & 1,317 (19.4\%) & $1,568(20.1 \%)$ & 0.34 \\
\hline$\beta$ Blockers & $1,230(18.1 \%)$ & $2,212(28.3 \%)$ & $<0.0001$ \\
\hline Calcium channel blockers & $528(7.8 \%)$ & 994 (12.7\%) & $<0.0001$ \\
\hline Anti-platelet (non-Aspirin) & $413(6.1 \%)$ & 549 (7.0\%) & 0.02 \\
\hline Statin & $1,499(22.1 \%)$ & $2,741(35.1 \%)$ & $<0.0001$ \\
\hline Warfarin & 395 (5.8\%) & $751(9.6 \%)$ & $<0.0001$ \\
\hline
\end{tabular}

MI, myocardial infarction; CAD, coronary artery disease; PVD, peripheral vascular disease; ACE-I, angiotensin-converting-enzyme inhibitor; ARB, angiotensin receptor blockers.

PET that make it most useful in screening for obstructive CAD $(11,12)$. Further, they have provided protocol guidelines for the use of PET imaging, thus overcoming a major barrier to its widespread adoption. However, little has been published assessing the outcomes after implementation of a PET-centered program compared with utilization of SPECT as the predominant MPI modality in a real-world setting.

In 2013, Intermountain Medical Center began to implement a PET-based program for MPI. To understand the differences between the 2-year period of SPECT utilization just before the PET program began, and the 2 years after PET was fully implemented, we conducted a retrospective analysis of prospectively collected catheterization outcomes 60 days after MPI and also assessed 1-year clinical outcomes to determine major adverse cardiovascular events (MACE) in the 2 periods. This represents 1 of the largest cohorts of PET-imaged patients reported to date. These findings may have broad implications as institutions consider the advantages and disadvantages of SPECT MPI and PET MPI.

\section{Results}

\section{Sixty-day catheter-based outcomes}

SPECT era vs. PET era. The demographic and clinical characteristics of patients for the patients in the PET era $(n=7,817)$ and the SPECT era $(n=6,777)$ are shown in Table 1 . There were statistically significant differences when comparing the PET and SPECT eras, given the large number of patients; however, most of the differences were small and not deemed to be clinically important (less than a $3 \%$ difference). The major exception was cardiovascular-related medication use, which was significantly higher in the PET compared with the SPECT era from both a statistical and clinical perspective.

To assess the post-stress test, catheter-based outcomes of the patients in the 2 different eras, we quantified the percentage of patients that underwent coronary angiography, the severity of the $C A D$, and whether 

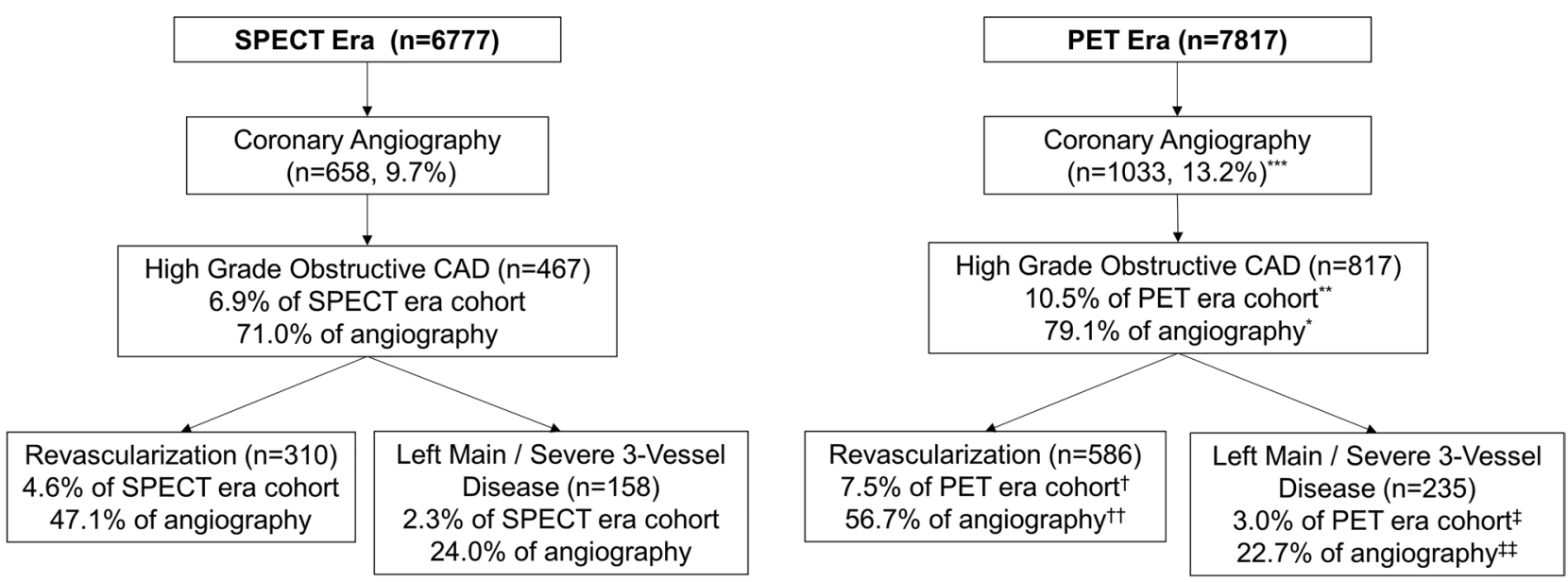

Figure 1. Sixty-day coronary catheterizations and outcomes of the SPECT and PET eras. Patients in the PET era had a higher rate of angiography, highgrade obstructive coronary artery disease (CAD), and revascularization than patients in the SPECT era. The percentage of left main and 3-vessel CAD was similar in the groups. Unadjusted $\chi^{2}$ tests were used to compare outcome percentages for the 2 eras (PET vs. SPECT).*** $P=3.6 \times 10^{-11}$ for 60 -day coronary angiography, ${ }^{* *} P=2.7 \times 10^{-14}$ for high-grade obstructive $C A D, \dagger P=1.4 \times 10^{-13}$ for revascularization, and $\ddagger P=0.012$ for left main or severe 3 -vessel disease. Restricting to patients that had an angiography, the $P$ values associated with percentage of outcomes for the 2 eras are ${ }^{*} P=1.8 \times 10^{-4}$ for high-grade obstructive $C A D, \dagger \dagger P=1.2 \times 10^{-4}$ for revascularization, and $\ddagger P=0.55$ for left main or severe 3 -vessel disease.

coronary revascularization was performed within the first 60 days after the index stress test (Figure 1). The percent of patients that underwent coronary angiography following a stress test was higher in the PET era compared with the SPECT era $(13.2 \%$ vs. $9.7 \%$, respectively, $P<0.0001)$. Importantly, high-grade obstructive CAD was identified more often in the PET era ( $10.5 \%$ vs. $6.9 \%$ for entire cohort, $P<0.0001$, and $79.1 \%$ vs. $71.0 \%$ for those undergoing angiography, $P<0.0001$ ). While left main or severe 3 -vessel disease were diagnosed more during the PET era compared with the SPECT, this was not statistically significant after adjustment for multiple testing (3.0\% vs. $2.3 \%$ for entire cohort, $P=0.012$, and $22.7 \%$ vs. $24 \%$ for those undergoing angiography, $P=0.55$ ). MPI during the PET era identified a higher percentage of the total number of patients undergoing a nuclear stress test that were deemed to need coronary revascularization $(7.5 \%$ vs. $4.6 \%$ for the entire cohort, $P<0.0001$, and $26.7 \%$ vs. $47.1 \%$ for those undergoing angiography, $P<0.0001$ ). The differences in 60 -day angiographic results during the PET era compared with the SPECT era were maintained even after adjustment for baseline differences in the populations in the 2 eras (Table 2 and Figure 2A).

SPECT test vs. PET test. As nearly $10 \%$ of the MPI stress tests done during the PET era were SPECT tests, we repeated the above analyses based on using those that had a SPECT rest and stress study during the SPECT era and those that had a PET test during the PET era. This resulted in 6,686 SPECT studies and 7,130 PET studies. More statically and clinically important differences in demographic and clinical characteristics were identified when restricting to type of test received (Supplemental Table 2; supplemental material available online with this article; https://doi.org/10.1172/jci.insight.120949DS1). The comparisons for the actual PET tests vs. SPECT tests were similar to the results for the era comparisons (Supplemental Table 3 and Supplemental Figure 1A).

Subgroups - MPI in patients without known CAD and outpatient MPI. We repeated the above analyses in a population without known atherosclerotic $\mathrm{CAD}$. In this screening population, there were higher rates of coronary angiography in the PET era when compared with the SPECT era (Table 3). Furthermore, after multivariable adjustment, coronary angiography after a stress test in the PET era was associated with nearly a 2-fold higher odds of identifying significant obstructive CAD (Table 3). Similarly, those that had been evaluated in the PET era were more than 2 times as likely to undergo coronary revascularization as those evaluated in the SPECT era (Table 3). Left main or severe 3-vessel disease was extremely uncommon in these screening populations for both eras (PET, $n=5$; SPECT, $n=3$ ).

Among the outpatient population (which includes those referred from the emergency department (ED), there was a higher rate of coronary angiography in the PET era compared with the SPECT era, a higher percentage of high-grade obstructive $\mathrm{CAD}$, and revascularization within 60 days (Table 3). 
Table 2. Sixty-day outcomes during PET and SPECT eras

\begin{tabular}{lccc}
\hline Sixty-day outcomes & PET era vs. SPECT era $n(\%)$ & Adjusted OR (95\% Cl) & P value \\
Coronary angiography & $1,033(13.2 \%)$ vs. $658(9.7 \%)$ & $1.47(1.32,1.64)$ & $<0.0001$ \\
High-grade CAD & $817(10.5 \%)$ vs. 467 (6.9\%) & $1.73(1.52,1.96)$ & $<0.0001$ \\
Left main/severe 3-vessel Disease & $235(3.0 \%)$ vs. 158 (2.3\%) & $1.45(1.17,1.80)$ & 0.0007 \\
Revascularization & $586(7.5 \%)$ vs. $310(4.6 \%)$ & $1.85(1.59,2.15)$ & $<0.0001$
\end{tabular}

Variables adjusted for included sex, race, BMI, family history of CVD, prior revascularization, history of heart failure, and medications (ACE or ARB, $\beta$ blocker, calcium channel blocker, anti-platelet, statin, and warfarin). OR, odds ratio.

These increases remained even after multivariable adjustment. Left main or severe 3-vessel disease was slightly higher in the PET era compared with the SPECT era, but due to small sample sizes, multivariable analyses were not done.

\section{Impact of MPI modalities on MACE}

SPECT era vs. PET era. There were a total of 450 (5.8\%) patients during the PET era that had a MACE-revascularization (MACE-revasc) event in the year following the imaging study, which was similar to the rate observed in the SPECT era (359 patients, 5.3\%) (Table 4). The demographic and clinical characteristics of the patients that had a MACE-revasc event during the 2 eras are provided in Supplemental Table 4, and the factors associated with MACE-revasc were similar for each era. The majority of the events were death in both eras (68.9\% of events for PET and $64.3 \%$ for SPECT). After adjusting for baseline and clinical characteristics, the rates of MACE-revasc was similar between the 2 eras (hazard ratio $[\mathrm{HR}]=1.08 ; 95 \%$ CI, 0.93-1.24; $P=0.31$ ) (Figure 2B). After adjustment, there were no differences in the rates of revascularization after 60 days (61-365 days), death, and myocardial infarction (MI) for the PET era versus the SPECT era (Table 4).

SPECT test vs. PET test. There were 403 (5.7\%) of the PET test patients that had a MACE-revasc after their stress imaging study, and this was similar to the rates for those patients that had a SPECT test $(n=347 ; 5.2 \%)(P=0.23)$. The majority of the events were deaths (PET test, $n=272,3.8 \%$, vs. SPECT test, $n=222,3.3 \%)$. After adjustment, there was still no significant difference in MACE-revasc events between the 2 groups ( $P=0.92)$ (Supplemental Figure 1B).

Subgroups - MPI in patients without known CAD and outpatient MPI. Among those with no known CAD, the MACE-revasc rates were about half the rate for the entire population cohorts for both the PET era $(n=93,2.4 \%)$ and the SPECT era $(n=132,3.0 \%)$. While there was a slight increase in the percentage of MACE-revasc for those patients without known CAD during the SPECT era compared with the PET era, this increase was not significant after adjustment for baseline differences $(P=0.24)$ (Table 3). Similarly, all patients evaluated with outpatient MPI had similar 1-year MACE-revasc rates during the PET era $(n=211,3.6 \%)$ and SPECT era $(n=174,3.5 \%)(P=0.14)$ (Table 3$)$.

\section{Discussion}

We examined the clinical outcomes of over 15,000 patients undergoing screening for ischemic cardiovascular disease, allowing for the largest sample size to date comparing PET and SPECT stress testing, to our knowledge. The results are from a real-world setting with pragmatic management and decision making. We found that a PET strategy compared with SPECT strategy resulted in increased rates of diagnosis of high-grade obstructive $\mathrm{CAD}$, left main/severe 3-vessel disease, and revascularization. As compared with the SPECT era, the PET era was associated with a lower incidence of invasive catheterization without identification of high-grade CAD.

While a higher percentage of patients that were evaluated with MPI during the PET era underwent coronary angiogram compared with the SPECT era, this does not necessarily indicate that utilization of PET increased the number of procedures performed. In fact, the total number of coronary angiographies (including angiographies without a prior MPI) decreased by $9.8 \%$ during the PET era $(n=5,103)$ compared with the SPECT era $(n=5,658)$. Thus, while the rate of patients having a coronary angiography following a PET is higher than the rate following a SPECT, this did not equate to an overall higher rate of coronary angiographies being performed at our institution (Intermountain Medical Center). Since there were fewer 


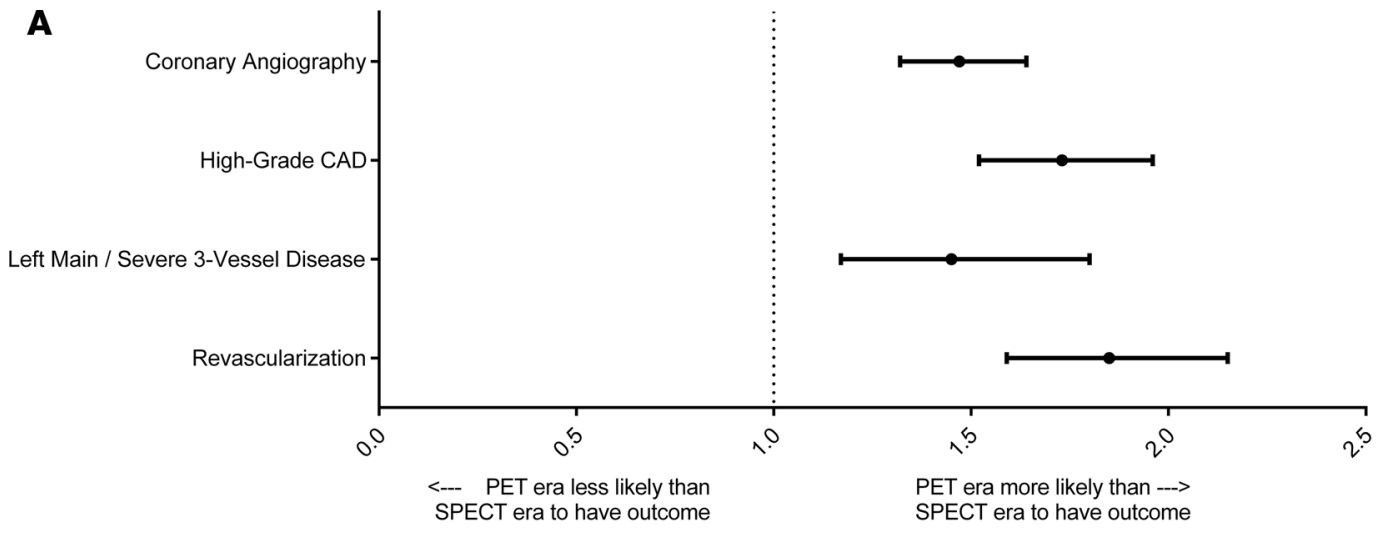

Adjusted OR for 60-day angiographic outcomes

B

MACE-Revasc. by Era

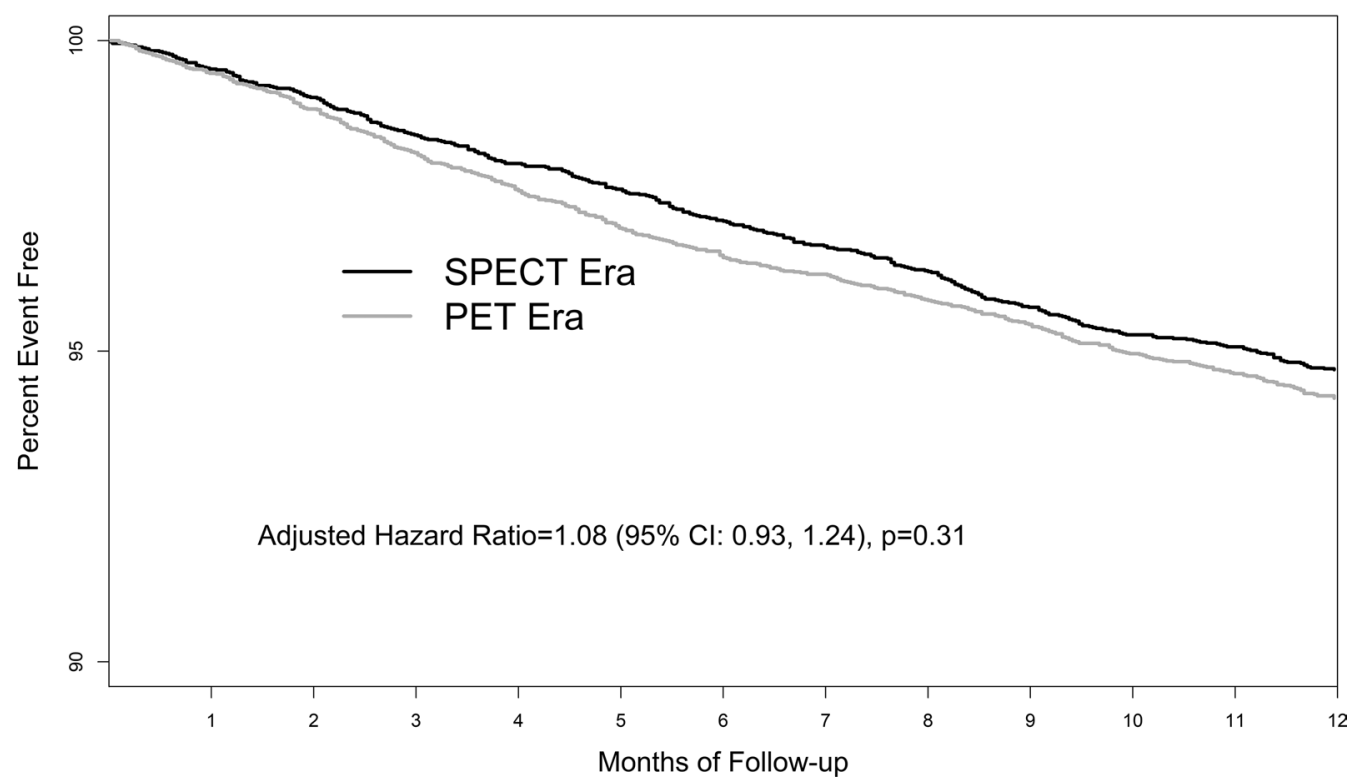

Figure 2. Adjusted odds ratio for the 60-day angiographic outcomes and the 1-year MACE-revascularization outcomes the PET era compared with SPECT era. After adjustment in a logistic regression model for baseline differences (sex, race, BMI, family history of CVD, prior revascularization, history of heart failure, and medications (angiotensin-converting-enzyme [ACE] or angiotensin receptor blockers [ARB], $\beta$ blocker, calcium channel blocker, anti-platelet, statin, and warfarin), patients in the PET era $(n=7,817)$ compared with SPECT era $(n=6,777)$ were more likely to have 60-day coronary angiography. High-grade obstructive coronary artery disease (CAD), left main or severe 3-vessel disease was identified more frequently in the PET era and associated with a higher rate of revascularization $(\mathbf{A})$. The rates of 1-year MACE-revascularization (including all-cause death, myocardial infarction, and post-60-day revascularization), after adjustment in Cox's proportional hazard model, were similar between the PET and SPECT eras (B).

total catheterizations performed during the PET era, it is also notable that a higher percentage of patients that underwent coronary angiography had been evaluated with an MPI study during the PET era than during the SPECT era (20.2\% vs. $12.9 \%)$.

Our experience contributes importantly to a growing understanding of the effect of implementation of a PET imaging program for detection of coronary ischemia in a real-world setting. Prior studies have shown similar strong performance of PET's diagnostic accuracy in different circumstances, with varying imaging protocols and study populations $(1,4,5,12-14)$. The recent head-to-head comparison between SPECT and PET of 208 Dutch patients with suspected CAD perhaps provides the most definitive evidence to date of the superior sensitivity of PET for detecting CAD (12). Our study, while observational in nature, provides catheterization and clinical outcomes on many more patients. PET imaging in both studies resulted in increased detection of high-grade $\mathrm{CAD}$, and while not significant, it did show a trend toward 
Table 3. Subgroup outcome analyses of screening population (those without prior MI, revascularization, and/or CAD) and outpatient/ ED population

\begin{tabular}{|c|c|c|c|}
\hline Subgroup outcomes & PET era vs. SPECT era $n(\%)$ & Adjusted OR/HR (95\% Cl) & $P$ value \\
\hline Screening population ${ }^{A}$ & $n=4,417$ vs. $n=3,833$ & & \\
\hline Sixty-day coronary angiography & 303 (6.9\%) vs. 200 (5.2\%) & $1.25(1.03,1.51)$ & 0.02 \\
\hline Sixty-day high-grade CAD & 157 (3.6\%) vs. 69 (1.8\%) & $1.94(1.44,2.60)$ & $<0.0001$ \\
\hline $\begin{array}{l}\text { Sixty-day left main/severe 3-vessel } \\
\text { disease }\end{array}$ & 5 (0.11\%) vs. 3 (0.08\%) & $N A^{B}$ & NA \\
\hline Sixty-day revascularization & 134 (3.0\%) vs. 53 (1.4\%) & $2.14(1.54,2.98)$ & $<0.0001$ \\
\hline Sixty-day coronary angiography & 618 (10.6\%) vs. 394 (7.9\%) & $1.37(1.19,1.58)$ & $<0.0001$ \\
\hline Sixty-day high-grade $C A D$ & $475(8.1 \%)$ vs. 269 (5.4\%) & $1.62(1.38,1.92)$ & $<0.0001$ \\
\hline $\begin{array}{l}\text { Sixty-day left main/severe 3-vessel } \\
\text { disease }\end{array}$ & 22 (0.38\%) vs. 14 (0.28\%) & $N A^{B}$ & NA \\
\hline Sixty-day revascularization & 343 (5.9\%) vs. 174 (3.5\%) & $1.84(1.51,2.23)$ & $<0.0001$ \\
\hline
\end{tabular}

${ }^{A}$ Variables adjusted for included sex, race, BMI, prior revascularization, history of heart failure, and medications (ACE or ARB, Aspirin, $\beta$ blocker, calcium channel blocker, anti-platelet, statin, and warfarin). ${ }^{B}$ Due to the small sample size, multivariable analyses and testing were not done. ${ }^{\mathrm{C}}$ Variables adjusted for included age, sex, race, BMI, prior revascularization, family history of CAD, and medications (ACE or ARB, Aspirin, $\beta$ blocker, calcium channel blocker, anti-platelet, statin, and warfarin).

increased identification of left main or severe 3-vessel CAD. This improvement might be due not only to improved imaging with PET, but also to the ancillary data available, such as myocardial blood flow and coronary calcium scores. An important consequence of this is that there are fewer coronary angiograms performed that did not result in revascularization using PET. As our patient population was generally similar between the 2 time periods, these findings may indicate that SPECT imaging fails to detect some highgrade obstructive CAD. Another explanation is that, when SPECT imaging is the only modality available, the cardiologist may opt to proceed directly to coronary angiography for those patients at greatest risk.

Our imaging protocols for PET are similar to those suggested for by the American Society of Nuclear Cardiology and Society of Nuclear Medicine and Molecular Imaging $(11,15)$. While these PET protocols have not been adequately evaluated and represent a gap in the current literature, the findings in our study do suggest that the current best-practice standards, which we applied, are associated with a high efficiency of identifying patients with high-grade $\mathrm{CAD}$.

The MACE event rates in the 2 patient cohorts were not significantly different. This adds further to the current debate on the optimal strategy for determining subsequent risk, or lack thereof, for MACE outcomes. The recent prospective multicenter imaging study for evaluation of chest pain (PROMISE), which compared anatomic imaging with coronary CT angiography (CTA) with traditional stress testing for the management of suspected CAD (16), also found no difference in MACE outcomes (death, myocardial infarction, hospitalization, or procedural complications) between strategies. This lack of significant differences in MACE outcomes was also reported in the Clinical Outcomes Utilizing Revascularization and Aggressive Drug Evaluation (COURAGE) trial, which found no decrease in MACE from percutaneous

Table 4. Major adverse cardiovascular events or revascularization (MACE-revasc) within 1 year for the SPECT era ( $n=6,777$ ) and PET era $(n=7,817)$

\begin{tabular}{lccc}
\hline One-year outcomes, entire population & PET era vs. SPECT era, $\boldsymbol{n}(\%)$ & Adjusted HR (95\% CI) & P value \\
MACE-revascularization & $450(5.8 \%)$ vs. $359(5.3 \%)$ & $1.08(0.93,1.24)$ & 0.31 \\
\hline Death & $310(4.0 \%)$ vs. $231(3.4 \%)$ & $1.15(0.96,1.37)$ & 0.12 \\
Hospitalization of MI & $77(1.0 \%)$ vs. $67(1.0 \%)$ & $1.01(0.73,1.42)$ & 0.94 \\
Revascularization (61-365 days) & $131(1.7 \%)$ vs. $107(1.6 \%)$ & $1.07(0.83,1.39)$ & 0.61
\end{tabular}


coronary intervention (PCI) compared with optimal medical therapy (17). Moreover, a meta-analysis of 12 similar randomized trials found no significant difference between PCI and medical therapy in relation to all-cause mortality, MI, and revascularization (18). Finally, a randomized trial of coronary CT screening of diabetic patients without a history of CAD also failed to show significant decreases in MACE (19). The MACE event rates in our MPI experience are consistent with these imaging-guided coronary ischemia trials. Therefore, our inability to detect significant differences in MACE outcomes between SPECT and PET in an observational study with limited follow-up time should not be surprising.

Limitations. There are inherent limitations in the observational design of this study. The 2 study groups were from 2 different time periods rather than randomly and/or concurrently assigned. This could result in temporal or other confounding differences between the groups. To examine this concern, baseline demographic and clinical characteristics were compared, and factors that differed significantly were included in an adjusted model for the outcomes. Nevertheless, one cannot entirely dismiss the possibility that unknown factors could have contributed to a portion of the observed differences. We noted no significant systematic changes in the indications for our approach to stress testing or coronary angiography that likely could have impacted the results. The majority of cardiologists reading and interpreting the scans were the same during the 2 time periods. Another limitation is the possibility of missing outcomes, as a patient may seek subsequent treatment outside an Intermountain facility or move out of state. The likelihood of this occurring is believed to be small, based on previous research of similar patient populations, and its impact would be expected to be similar in both the SPECT and PET cohorts. This study was based on a single institution design, with findings that may not translate equally to all institutions, particularly those of different sizes, population characteristics, or diagnostic strategies. Furthermore, our study does not address a comprehensive evaluation of the institutional costs that result from transition from a mainly SPECT- to a PET-based MPI strategy. However, it is notable that there was a decrease in the total number of diagnostic catheterizations performed in our medical system in the PET era compared with the SPECT era.

Conclusion. The use of PET MPI, compared with SPECT MPI, as a primary method of myocardial ischemia detection was associated with a significant increase in the detection of high-grade obstructive $\mathrm{CAD}$ and resulted in higher rates of revascularization in the first 60 days. This did not result in a significant decrease in the rate of 1-year MACE, which is consistent with findings of past clinical MPI-guided trials that show differences in symptom relief but not in MACE following revascularization. We are encouraged by these results but realize that further refinement of protocols and best practices for the implementation and interpretation of PET MPI testing, additional prospective trials, and the publishing of results will be critical to facilitating more widespread adoption of PET MPI.

\section{Methods}

Study population. This was a retrospective observational study. Patient populations from 2 time periods were studied - data were collected on consecutive MPI patients at Intermountain Medical Center from January 1, 2011, to December 31, 2012 (the SPECT era, $n=6,777$ ), and consecutive MPI patients at Intermountain Medical Center from January 1, 2014, to December 31, 2015 (the PET era, $n=7,817$ ). During the SPECT era, patients were evaluated with both a rest and stress exam, and during times of overflow, some MPI during the PET era was done using SPECT. We chose to compare outcomes between the PET and SPECT eras in 2 ways: the first and primary analyses compared all MPI patients during the 2 eras regardless of the MPI modality used (an intention-to-treat approach), and the second and supplementary analyses compared SPECT patients during the SPECT period, who received both a rest and a stress test, with the PET patients during the PET era (a per-protocol approach). If a person had more than 1 MPI, the first test was used for analyses. As we wanted to examine subsequent events and outcomes, we excluded any patient who died prior to discharge and any patient who was discharged to hospice with a subsequent death within 60 days.

SPECT era. Almost half of the studies done in the SPECT era were pharmacologic stress studies $(n=$ $3,333,49.2 \%)$. The pharmacologic agent of choice was regadenoson $(n=3,327,99.8 \%)$. A quarter of the SPECT tests were exercise SPECT tests $(n=1,693,25.0 \%)$, and another quarter were a combination of exercise supplemented with pharmacologic agent due to failure to achieve target heart rate thresholds $(n$ $=1,660,24.5 \%)$. A few of the SPECT era tests were rest only $(n=89,1.3 \%)$ or stress only $(n=2,<0.1 \%)$ (Supplemental Table 1).

SPECT myocardial perfusion images were acquired as part of a routine rest-stress protocol using 99mTc-tetrofosmin (Tc99m) with a few rest-only and stress-only exceptions, as noted previously. The 
weight-based Tc99m dosage varied from 9-12 mCi for rest and 28-34 mCi for stress imaging. The cardiac SPECT studies were performed on a GE Hawkeye 4-slice CT camera. The following camera parameters were used: low-energy high-resolution collimator, 30-60 minute rest and 15-60 minute stress injection to imaging time; time to projection, 25 seconds for rest and 20 seconds for stress with Matrix 64, with stress-gated 8 frames/cycle and filtered back projection (FBP) reconstruction. CT attenuation correction was performed on rest and stress with nondiagnostic CTs.

PET era. Intermountain Medical Center switched to the use of PET in March 2013; as 2013 was a transitional year between SPECT and PET, it was not included in these analyses. Even after widespread adoption of PET in 2014 and 2015 (PET era), there were a few patients that underwent SPECT MPI $(n=680,8.7 \%)$ and a few with rest-only studies $(n=59,0.8 \%)$ (Supplemental Table 1$)$. Most of the SPECT tests were during overflow times for PET. During the PET era, PET was used regardless of the ability for a patient to do exercise SPECT.

PET imaging was performed on a Siemens Biograph (LSO crystal, 3-dimensional-list mode, 16-slice $\mathrm{CT})$ camera with rubidium- 82 chloride $\left({ }^{82} \mathrm{Rb}\right.$ chloride). Weight-based ${ }^{82} \mathrm{Rb}$ dosage varied from $30-40 \mathrm{mCi}$ for both rest and stress images. CT images were reviewed for presence of coronary calcium. Patients were injected under the PET camera for immediate macromolecular proton fraction (MBF) imaging with list mode acquisition for 7 minutes. Pharmacologic stress was achieved in all patients with regadenason. Both gated rest and stress images were acquired and iteratively reconstructed using the manufacturer recommended protocol.

The reading and interpretation of both types of MPI studies during the study timeframe were performed by the same group of cardiologists, all of whom were board-certified in nuclear cardiology. Both the SPECT and PET MPI images were analyzed using commercially available software packages (QPS/ QGS; Cedars-Sinai Medical Center and Corridor4DM, Invia Medical Imaging Solutions).

Endpoints. Five primary outcomes were studied: (a) coronary artery angiography within 60 days of the stress test, (b) the reporting of high-grade obstructive CAD ( $\geq 70 \%$ stenosis) within 60 days of the stress test, (c) left main or severe 3-vessel disease diagnosis within 60 days of the stress test, (d) revascularization (PCI and/or coronary artery bypass grafting [CABG]) within 60 days of the stress test, and (e) 1-year MACE (MACE-revasc), which included death, hospitalization for MI, or post-60-day revascularization. The reporting of death was determined using in-hospital deaths and deaths reported to the Utah Department of Health through death certificates. As the deaths for all Utah residents and deaths within the state of Utah are required to be reported to the Utah Department of Health, we are confident that we have captured nearly all deaths among the study population. The nonfatal outcomes were determined by searching Intermountain Healthcare's electronic data warehouse using diagnosis and procedure codes. The electronic data warehouse contains all diagnosis and procedures occurring at any of the 22 hospitals and over 180 clinics within the Intermountain Healthcare integrated system. As we are examining outcomes within a year and most patients are not likely to have changes in treating physicians, facility, or insurances during this period, we again feel like we have captured nonfatal events to a degree greater than $90 \%$. In fact, in a nonpublished survey of our patients, we found that over $90 \%$ reported receiving all subsequent cardiac care at an Intermountain facility. To rule out miscoding of MI associated with the index MPI evaluation or subsequent coronary angiography-related troponin elevations, hospitalizations for MI within 60 days of testing were excluded if the troponin was less than $2 \mathrm{ng} / \mathrm{ml}$.

Statistics. The baseline demographic and clinical characteristics were compared between the 2 modality eras using $\chi^{2} /$ Fisher's exact test (for categorical values) and Wilcoxon rank sum tests (for continuous values). The demographic and clinical characteristics that were significantly different were adjusted for by multivariable modeling of outcomes. The modeling of the 60-day outcomes was done using logistic regression. The 1-year MACE-revasc outcome, which included death, MI, and post-60day revascularization, was modeled by time-to-event analysis with Cox proportional hazards regression. To account for multiple testing, significance was set at $P<0.01$ for the 5 primary outcomes. A nominal significance levels of $P<0.05$ was used for the additional secondary and subgroup analyses. SAS 9.3 was used for all the analyses.

Study approval. This retrospective observational study was approved by the Intermountain Healthcare IRB in Murray, Utah, USA, and was given a waiver of consent, as this was a minimal-risk retrospective study using existing data. Investigations were in accordance with the Declaration of Helsinki. 


\section{Author contributions}

SK contributed design, data analyses, and manuscript preparation. DBM, KGM, RD, SB, SMM, JDE, and KRJ contributed design, data collection, data analysis interpretation, and manuscript preparation/ review. VTL, DLL, JBM, JLA, and KUK contributed design, data analysis interpretation, and manuscript preparation/review.

Address correspondence to: Kirk U. Knowlton, Director of Cardiovascular Research, Associate Chief of Cardiology, Intermountain Heart Institute, 5121 South Cottonwood Street, Salt Lake City, Utah 84107-5701, USA. Phone: 801.507.4757; Email: kirk.knowlton@imail.org.

1. Kaster T, Mylonas I, Renaud JM, Wells GA, Beanlands RS, deKemp RA. Accuracy of low-dose rubidium- 82 myocardial perfusion imaging for detection of coronary artery disease using 3D PET and normal database interpretation. J Nucl Cardiol. 2012;19(6):1135-1145.

2. Di Carli MF, Dorbala S, Meserve J, El Fakhri G, Sitek A, Moore SC. Clinical myocardial perfusion PET/CT. J Nucl Med. 2007;48(5):783-793.

3. Leccisotti L, Lavalle M, Giordano A. Clinical relevance of PET myocardial blood flow quantification. Q J Nucl Med Mol Imaging. 2016;60(4):338-353.

4. Bateman TM, et al. Diagnostic accuracy of rest/stress ECG-gated Rb-82 myocardial perfusion PET: comparison with ECG-gated Tc-99m sestamibi SPECT. J Nucl Cardiol. 2006;13(1):24-33.

5. Sampson UK, Dorbala S, Limaye A, Kwong R, Di Carli MF. Diagnostic accuracy of rubidium-82 myocardial perfusion imaging with hybrid positron emission tomography/computed tomography in the detection of coronary artery disease. $J \mathrm{Am} \mathrm{Coll}$ Cardiol. 2007;49(10):1052-1058.

6. Jaarsma C, et al. Diagnostic performance of noninvasive myocardial perfusion imaging using single-photon emission computed tomography, cardiac magnetic resonance, and positron emission tomography imaging for the detection of obstructive coronary artery disease: a meta-analysis. J Am Coll Cardiol. 2012;59(19):1719-1728.

7. Nandalur KR, Dwamena BA, Choudhri AF, Nandalur SR, Reddy P, Carlos RC. Diagnostic performance of positron emission tomography in the detection of coronary artery disease: a meta-analysis. Acad Radiol. 2008;15(4):444-451.

8. Ghotbi AA, Kjaer A, Hasbak P. Review: comparison of PET rubidium-82 with conventional SPECT myocardial perfusion imaging. Clin Physiol Funct Imaging. 2014;34(3):163-170.

9. Takx RA, et al. Diagnostic accuracy of stress myocardial perfusion imaging compared to invasive coronary angiography with fractional flow reserve meta-analysis. Circ Cardiovasc Imaging. 2015;8(1):e002666.

10. Mc Ardle BA, Dowsley TF, deKemp RA, Wells GA, Beanlands RS. Does rubidium-82 PET have superior accuracy to SPECT perfusion imaging for the diagnosis of obstructive coronary disease?: A systematic review and meta-analysis. $J$ Am Coll Cardiol. 2012;60(18):1828-1837.

11. Bateman TM, Dilsizian V, Beanlands RS, DePuey EG, Heller GV, Wolinsky DA. American Society of Nuclear Cardiology and Society of Nuclear Medicine and Molecular Imaging Joint Position Statement on the Clinical Indications for Myocardial Perfusion PET. J Nucl Med. 2016;57(10):1654-1656.

12. Danad I, et al. Comparison of Coronary CT Angiography, SPECT, PET, and Hybrid Imaging for Diagnosis of Ischemic Heart Disease Determined by Fractional Flow Reserve. JAMA Cardiol. 2017;2(10):1100-1107.

13. Marwick TH, Shan K, Patel S, Go RT, Lauer MS. Incremental value of rubidium-82 positron emission tomography for prognostic assessment of known or suspected coronary artery disease. Am J Cardiol. 1997;80(7):865-870.

14. Parker MW, et al. Diagnostic accuracy of cardiac positron emission tomography versus single photon emission computed tomography for coronary artery disease: a bivariate meta-analysis. Circ Cardiovasc Imaging. 2012;5(6):700-707.

15. Dilsizian V, et al. ASNC imaging guidelines/SNMMI procedure standard for positron emission tomography (PET) nuclear cardiology procedures. J Nucl Cardiol. 2016;23(5):1187-1226.

16. Douglas PS, et al. Outcomes of anatomical versus functional testing for coronary artery disease. $N$ Engl J Med. 2015;372(14):1291-1300.

17. Boden WE, et al. Optimal medical therapy with or without PCI for stable coronary disease. NEngl J Med. 2007;356(15):1503-1516.

18. Pursnani S, et al. Percutaneous coronary intervention versus optimal medical therapy in stable coronary artery disease: a systematic review and meta-analysis of randomized clinical trials. Circ Cardiovasc Interv. 2012;5(4):476-490.

19. Muhlestein JB, et al. Effect of screening for coronary artery disease using CT angiography on mortality and cardiac events in high-risk patients with diabetes: the FACTOR-64 randomized clinical trial. JAMA. 2014;312(21):2234-2243. 